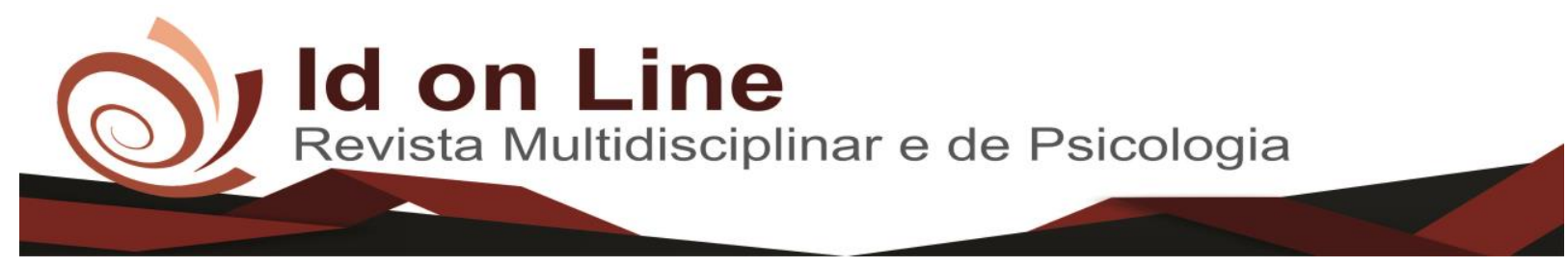

Relato de Caso

\title{
Montagem de Prótese Total em Paciente com Mordida Cruzada: Relato de Caso Clínico
}

\author{
Rogério Nogueira Celino Carneiro ${ }^{1}$; Jônatas Pereira do Prado ${ }^{2}$; \\ Miguel Arcanjo Porto da Cunha ${ }^{3}$; Daniela Porto da Cunha ${ }^{4}$
}

\begin{abstract}
Resumo: A perda de um ou mais elemento dental reflete diretamente na vida de um indivíduo, desde dificuldade em se alimentar, vergonha e prejuízo ao relacionamento social, tornando-se peça fundamental no desenvolvimento de uma Odontologia mais conservadora e a mais reparadora possível. Nesse sentido, a reabilitação dental se liga direta ou indiretamente a estética, fazendo com que haja uma atenção aumentada, e aliada a esta, a função e o conforto são fatores que necessitam ser considerados para alcançar o sucesso de um tratamento. Na tentativa de buscar um equilíbrio neuromuscular do sistema estomatognático, a reabilitação de pacientes edêntulos por meio de próteses dentárias auxilia de modo que suas funções sejam recuperadas e que o bem-estar físico, mental e social do paciente seja alcançado. Dessa forma a construção de próteses individualizadas para cada paciente se torna peça chave no sucesso de um tratamento reparador. Assim, este trabalho tem por objetivo relatar um caso clínico no qual houve a confecção de próteses totais individualizadas à um caso particular de um indivíduo que apresentava mordida cruzada.
\end{abstract}

Palavras-chave: Prótese Dentária; Má Oclusão; Reabilitação Bucal.

\section{Assembly Of Total Protesis In Patient With Crossbite: A Clinical Case Report}

\begin{abstract}
The loss of one or more dental elements reflects directly on an individual's life, from difficulty in feeding, shame and loss to social relationships, becoming a fundamental part of the development of a more conservative and restorative dentistry. In this sense, dental rehabilitation is directly or indirectly linked to aesthetics, leading to increased attention, and allied to this, function and comfort are factors that need to be considered to achieve the success of a treatment. In an attempt to achieve a neuromuscular balance of the stomatognathic system, the rehabilitation of edentulous patients by means of dental prostheses helps so that their functions are recovered and that the patient's physical, mental and social well-being is achieved. In this way, the construction of individualized prostheses for each patient becomes a key part in the success of a restorative treatment. Thus, this study aims to report a clinical case in which individual dentures were made to a particular case of an individual presenting with crossbite.
\end{abstract}

Keywords: Dental Prosthesis; Malocclusion; Oral Rehabilitation.

\footnotetext{
${ }^{1}$ Graduando do Curso de Odontologia da Faculdade Independente do Nordeste-FAINOR.

Contato: rogerio_celino@ hotmail.com

${ }^{2}$ Graduando do Curso de Odontologia da Faculdade Independente do Nordeste-FAINOR

${ }^{3}$ Docente do Curso de Odontologia da Universidade Estadual da Bahia - UESB/ Docente do Curso de Odontologia da Faculdade Independente do Nordeste-FAINOR

${ }^{4}$ Docente do Curso de Odontologia da Faculdade Independente do Nordeste-FAINOR
} 


\section{Introdução}

A perda total dos dentes dentre as situações clínicas da Odontologia é uma das piores, vez que gera diminuição da capacidade mastigatória e interferindo na digestão alimentar, constituindo um fator que afeta nas relações sociais, podendo gerar isolamento, prejudicando a qualidade de vida do paciente, afetando ainda a fonação e a estética. ${ }^{1}$

Reconhece-se hoje, que os progressos tecnológicos trouxeram melhorias para a saúde e na Odontologia não seria diferente, os padrões de saúde geraram um aumento significativo na expectativa de vida e idosos atualmente vivem bem melhor. ${ }^{2}$ Muito embora a saúde bucal da população idosa no Brasil ainda passe por problemas, que são frutos do modelo assistencial arcaico centralizado em práticas curativas e mutiladoras, o que gerou ausência de dentes em uma grande parcela populacional e ainda uma ampla necessidade de serviços protéticos por parte dessa população. ${ }^{3}$

Conforme levantamentos epidemiológicos, realizados em 2010 a necessidade de prótese foi observada no inquérito populacional em um número muito significativo de idosos com necessidade de reabilitação protética. ${ }^{4}$ Com base em tais preocupações, a reposição dos dentes por meio de próteses prima por trazer maior qualidade de vida ao idoso, proporcionando-lhes retorno à condição de vida e dando-lhes um melhor padrão estético e funcional. ${ }^{5}$

E no caso de mordida cruzada, a técnica de montagem de dentes numa prótese total é um recurso aplicado desde o século XIX, quando o dentista Alfred Gysi utilizava montagem de dentes primando trazer uma melhor eficiência mastigatória criando assim dentes específicos para mordida cruzada, levando em consideração a anatomia e inclinação dos dentes artificiais para oclusão posterior normal. , $^{6}$

$\mathrm{Na}$ tentativa de buscar um equilíbrio neuromuscular do sistema estomatognático, a reabilitação de pacientes edêntulos por meio de próteses dentárias auxilia de modo que suas funções sejam recuperadas e que o bem-estar físico, mental e social do paciente seja alcançado. ${ }^{5}$ Para tanto, ela deve buscar manter os traços faciais típicos, o perfil, a forma e suporte dos lábios e a harmonia ao sorrir, resultando numa fisionomia rotulada como agradável. O primordial é alcançar um sorriso harmonioso com os atributos próprios de cada paciente. ${ }^{8}$

$\mathrm{Na}$ confecção de próteses totais, o cirurgião-dentista deve primar por restabelecer a forma e a função do sistema estomatognático, restabelecendo a forma e função e tentar trazer 
uma aparência mais natural possível e o mais confortável ao paciente. ${ }^{4}$ Contudo, as vezes não é possível atingir todos os objetivos de uma reabilitação oral, tais como, uma boa retenção, estabilidade, estética e adequada fonação. Por isso que é necessário uma minuciosa anamnese somada a criterioso exame clínico e complementar para gerar maior êxito nas reabilitações. ${ }^{9}$

Importante ainda que o paciente entenda o tempo de adaptação às próteses, sendo salutar que o profissional incentive-os a utilizá-las e assim reduza qualquer tipo de desconfortos. ${ }^{10}$ Por isso é de extrema importância que seja dado destaque ao caso clínico explicitado, uma vez os dados apresentados podem representar uma possibilidade de disponibilização ao meio acadêmico e ao Cirurgiões-Dentistas subsídios para estudos posteriores, bem como o desenvolvimento de técnicas e materiais mais adequados para os casos desse tipo.

Por fim, vale destacar que o objetivo desse estudo é de apresentar, por meio de um relato de caso clínico, a montagem de dentes em próteses totais de um indivíduo que possui uma mordida cruzada posterior bilateral.

\section{Caso Clínico}

Paciente E.B.S, 66 anos, participou do tratamento em 12 sessões. A primeira foi feita a anamnese, exame clínico intra oral e extra oral; com fotografias iniciais e a solicitação de exame complementar (panorâmica). Na Anamnese contatou-se que ele usava prótese total desde os 16 anos e realizou exodontia de todos elementos dentários devido lesões cariosas e entre seus problemas de saúde foram citados colesterol, pressão alta, cardiopata.

No exame extra-oral foi analisado a NDN e no e exame intra-oral, foi averiguado no lábio: grânulos de Fordayce; queilite angular; na mucosa jugal: marcas de mordeduras; nos rebordos: inferior pouco, já o superior um pouco satisfatório, exostose em palato devido câmara de sucção em prótese; no assoalho: NDN, na língua: saburra;, verificando mandíbula volumosa, tendendo à uma classe III funcional; percebendo a má oclusão tida como mordida cruzada posterior e a maxila muito atrésica, tendo como procedimento realizado o exame clínico intra e extra oral, fotografias, solicitação de exame complementar (panorâmica).(Figura 1) 


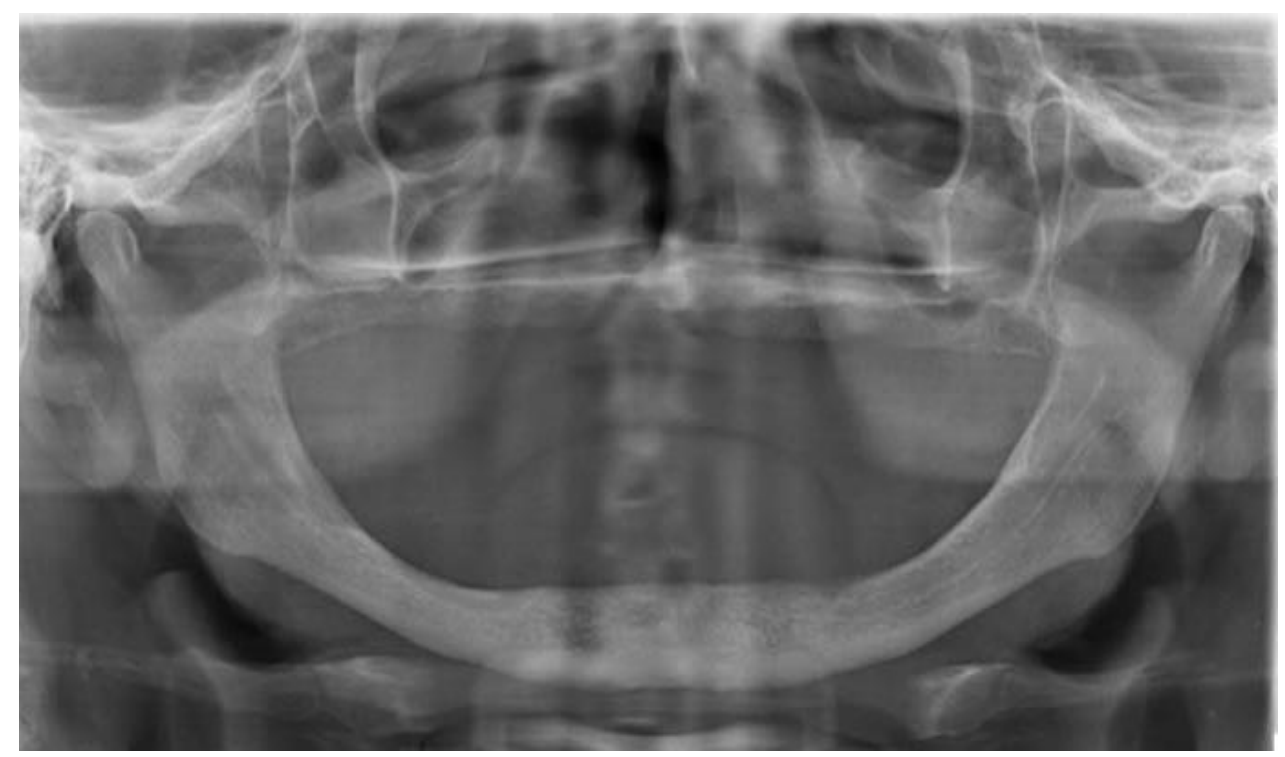

Figura 1: Referente a sessão: 1 resultado da panorâmica

A $2^{\mathrm{a}}$ sessão foi feita a visualização do exame complementar; moldagem anatômica; inserção da pasta lysanda na câmara de sucção e o modelo anatômico. Observando se a moldeira estar adaptada, cobrindo a região do trigoretromolar, sendo selecionada a moldeira 3 e a seleção da moldeira superior. Vale acrescer que foi necessária utilização de anestésico tópico $\left(\right.$ Benzotop $^{\circledR}$ ) na região do palato para diminuir a ânsia de vômito. Após a seleção foi utilizado a cera periférica tanto na moldeira superior como na inferior. Sendo que na superior foi colocado a cera na região central da moldeira evitando assim o excesso nessa região (palato).

Em seguida foi feita a manipulação do alginato (aglutinado e não misturado) na proporção: 3 de pó e 2,5 de água e em seguida foi feita a moldagem. O paciente sentou- se na cadeira de operações, estando ligeiramente inclinada para trás e sua cabeça apoiada no encosto. O profissional solicitou a ele que respirasse pelo nariz. O profissional deve pedir ao paciente que movimente a lima para cima e na direção da comissura labial para direita e esquerda.

Após moldagem verificou-se que o molde não apresentava bolhas e que as regiões anatômicas foram copiadas corretamente; Como na prótese que o paciente utiliza há presença de uma câmara de sucção, e houve promoção de uma exostose, nessa sessão foi colocada pasta Lysanda ${ }^{\circledR}$, afim de que houvesse diminuição por compressão do toros. Por fim fez-se o vazamento do molde, para obtenção do modelo anatômico, com gesso tipo II. 
$\mathrm{Na}$ terceira sessão (Figura 2) foi feito o recorte do modelo e a moldeira individual, seguida pela quarta que fez o vedamento periférico e a quinta que fez a moldagem funcional; encaixotamento e o modelo funcional. A moldagem da arcada inferior com pasta Lysanda®, vaselinou-se região de vermelhão do lábio, usando os seguintes materiais: placa de vidro forrada, espátula para manipulação, moldeira individual. Nessa oportunidade o paciente não deve fechar, nem abrir a boca com muito esforço e sim, relaxar o máximo possível, porque o arco ósseo mandibular costuma fechar quando o paciente faz esforços. Tracionando a mucosa jugal para frente, visa-se o registro da impressão na borda mais posterior do arco retrovestibular e o tempo de presa do material utilizado: 3 a 5 minutos, a depender da temperatura. Para realizar limpeza do excesso de material no paciente, utiliza-se o líquido da resina acrílica.

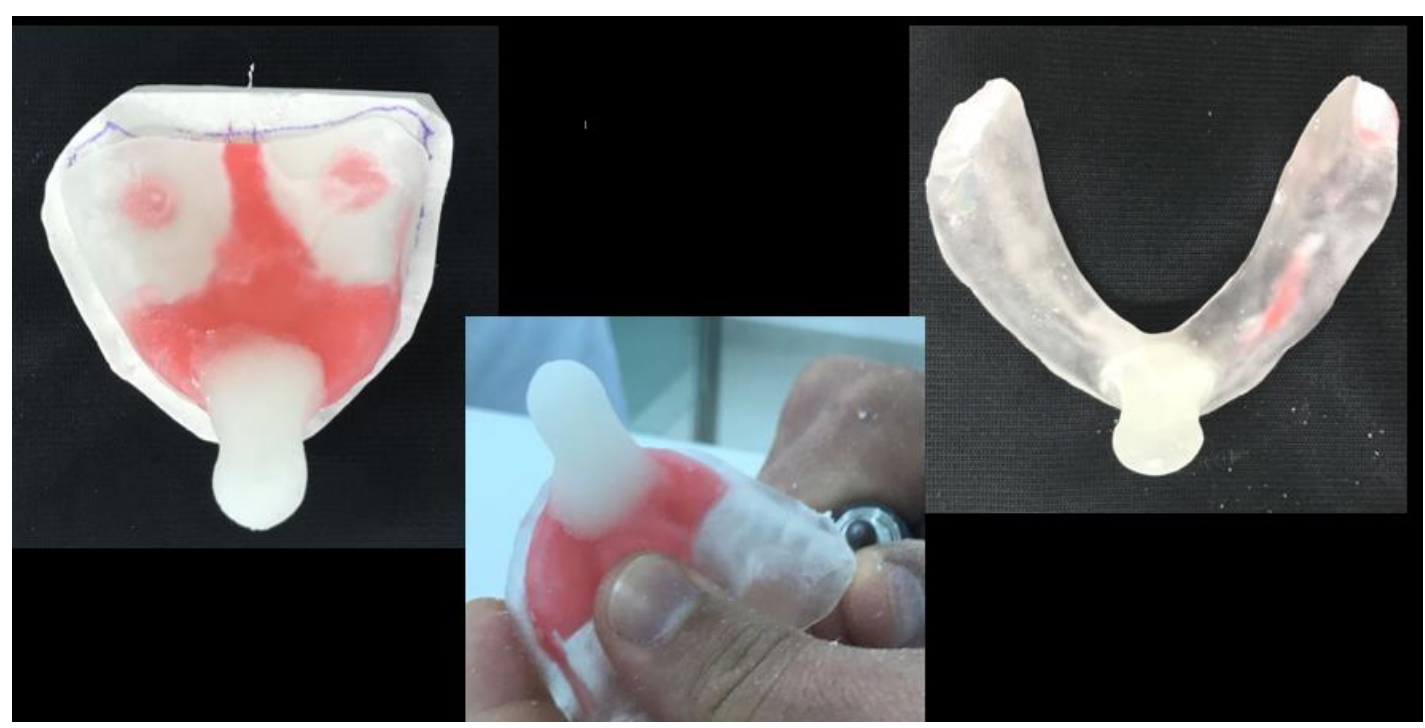

Figura 2: Referente à $3^{\mathrm{a}}$ sessão

Para a moldagem superior foi feita aplicação do adesivo na parte interna da moldeira individual (bacia da moldeira), manipulação do material e preencheu a moldeira com Impregum ${ }^{\circledR}$. Após a obtenção da estabilização da moldeira, coloca-se o indicador apoiado firmemente na abóboda palatina e pede-se ao paciente para que faça o movimento como se quisesse 'morder' o dedo do operador com os lábios. Essa ação faz baixar o sulco gengiovestibular, e deixar sua impressão nas bordas e tracionando a mucosa jugal para cima, para fora e suavemente para baixo e para trás, evidenciando-se a obtenção do lateral e do arco 
zigomático, já tracionando a mucosa jugal para trás e para frente, visa obter a impressão do arco da tuberosidade na borda mais posterior por vestibular (Figura 3).

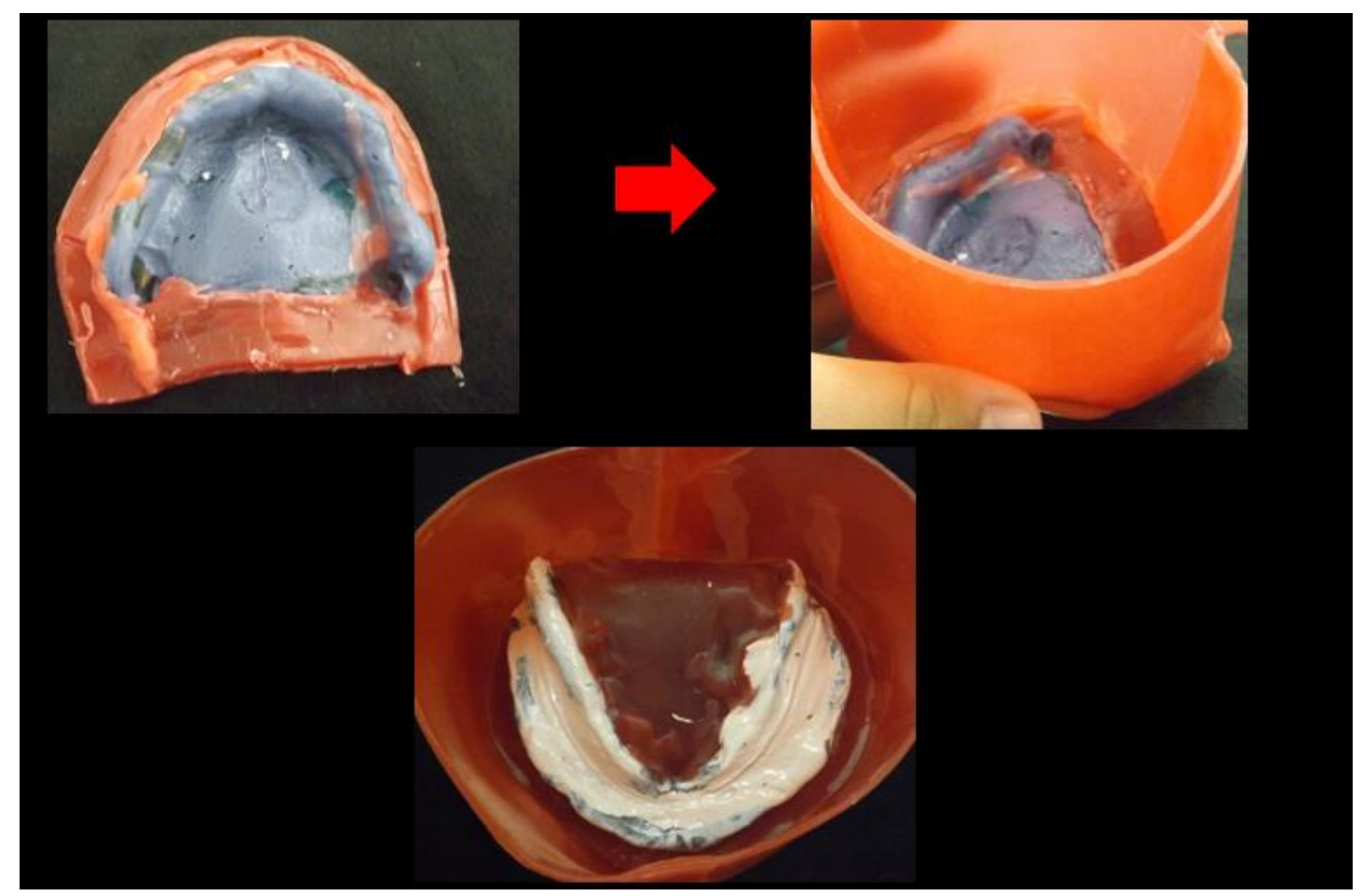

Figura 3: Referente à $5^{\mathrm{a}}$ sessão

Na sexta sessão fez-se a remoção da cera do encaixotamento; e remoção dos modelos dos moldes. Na moldeira em que estava a Lysanda® a remoção foi mais difícil a remoção do modelo. Sendo necessário mergulhar o conjunto em água quente (usa-se o aquecedor). Devido a este aquecimento, a godiva presente no vedamento periférico aderiu-se ao modelo, sendo utilizada lecron e espátula e para remoção desses resíduos e foi feita a confecção da base de prova. Os modelos superior e inferior foram preparados e isolados, pincelando-se líquido isolante, para que a resina acrílica não se adira ao gesso, sendo feita inserção da resina acrílica, por meio da técnica pó/líquido.

A sétima sessão foi utilizada cera utilidade, dobradas, tanto em arcada superior como inferior. Ao ser aquecida foi dada a forma de cada arcada, preconizando o que está na literatura (superior: $20 \mathrm{~mm}$ em região anterior e $5 \mathrm{~mm}$ em região posterior; e inferior: $18 \mathrm{~mm}$ em região 
anterior e 0 em região posterior), com auxílio da curva de spee, para posterior adaptação em bocas do paciente.

No oitavo encontro foi realizado ajuste no plano de cera, após teste em boca do paciente. Constatou-se uma perda de dimensão vertical de oclusão, onde foi devolvida em plano de cera (em prótese antiga $\mathrm{DVR}=48 \mathrm{~mm} / \mathrm{DVO}=51 \mathrm{~mm}$ e com a base $\mathrm{DVR}=60 \mathrm{~mm} / \mathrm{DVO}=63 \mathrm{~mm})$. Confecção de canaletas e confecção de canaletas para moldar uma área correspondente ao espaço funcional livre.

Na nona sessão (Figura 4) fez-se a montagem em Articulador realizada em relação cêntrica. Primeiro montou-se a arcada superior, com auxílio do plano de camper, e posteriormente a arcada superior, sendo feito o recorte do modelo em asa e o envio ao laboratório para montagem dos dentes em plano de cera.

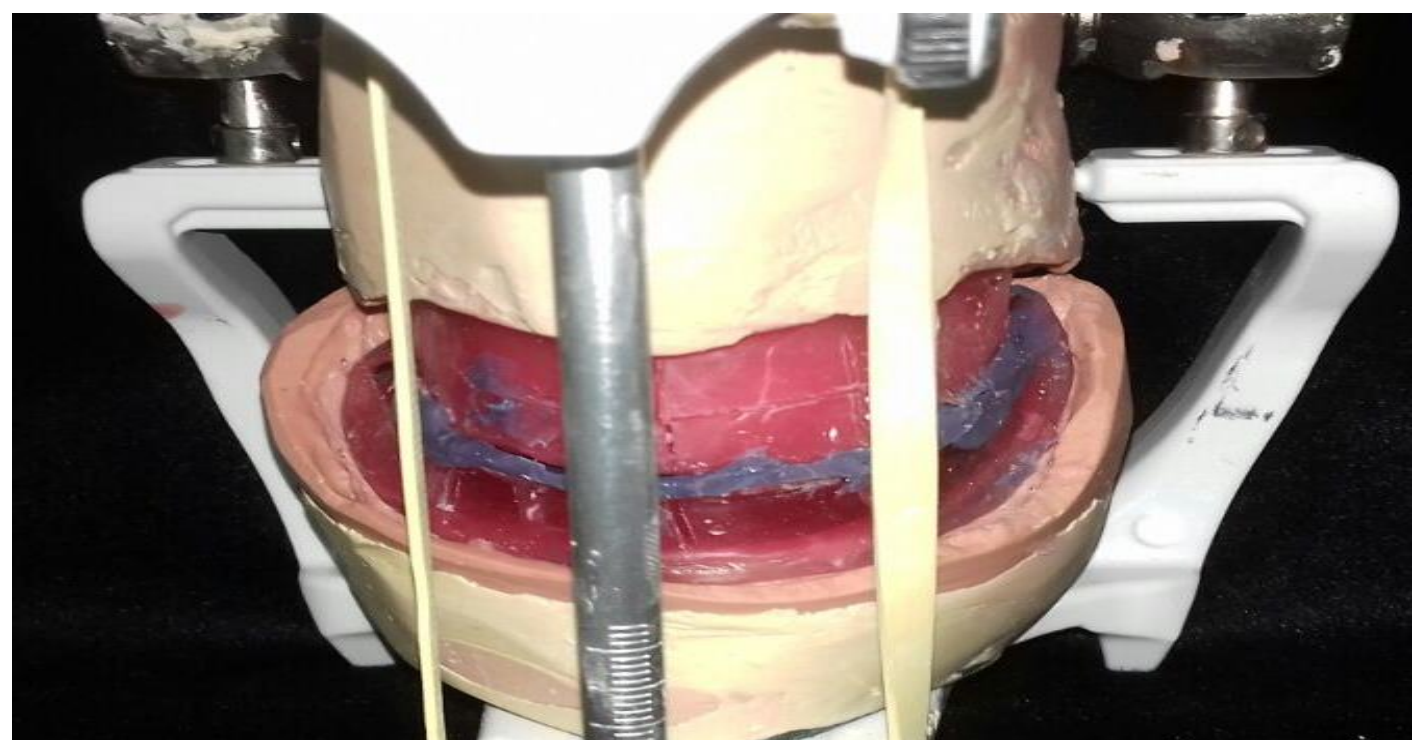

Figura 4: Referente à $9^{a}$ sessão

Na decima sessão foi a prova dos dentes montados no plano de cera e nesta constatou que a linha média inferior não coincidia com a superior e havia lingualização dos incisivos inferiores, contudo em ASA estava tudo certo e foram feitos desgastes na mesma região da peça inferior e houve novo teste em paciente, onde foi possível observar que ocorreu o ajuste da linha média e a oclusão estava certa. Foi realizada nova moldagem do arco superior, com 
Impregum®. Foi feita a seleção de cor da gengiva: 16 ou A5 e foram enviados para o laboratório para serem acrilizados, tanto superior como inferior.

Na decima primeira sessão foi realizado prova da prótese já acrilizada sem necessidade de ajustes. (Figura 5) E na ultima sessão (número 12) foi feito o ajuste oclusal com auxílio de um carbono, no qual foi realizada marcações na PT nova, em movimentos cêntricos e excêntricos, e realizado um pequeno ajuste oclusal. Finalizando o tratamento.(Figura 6)

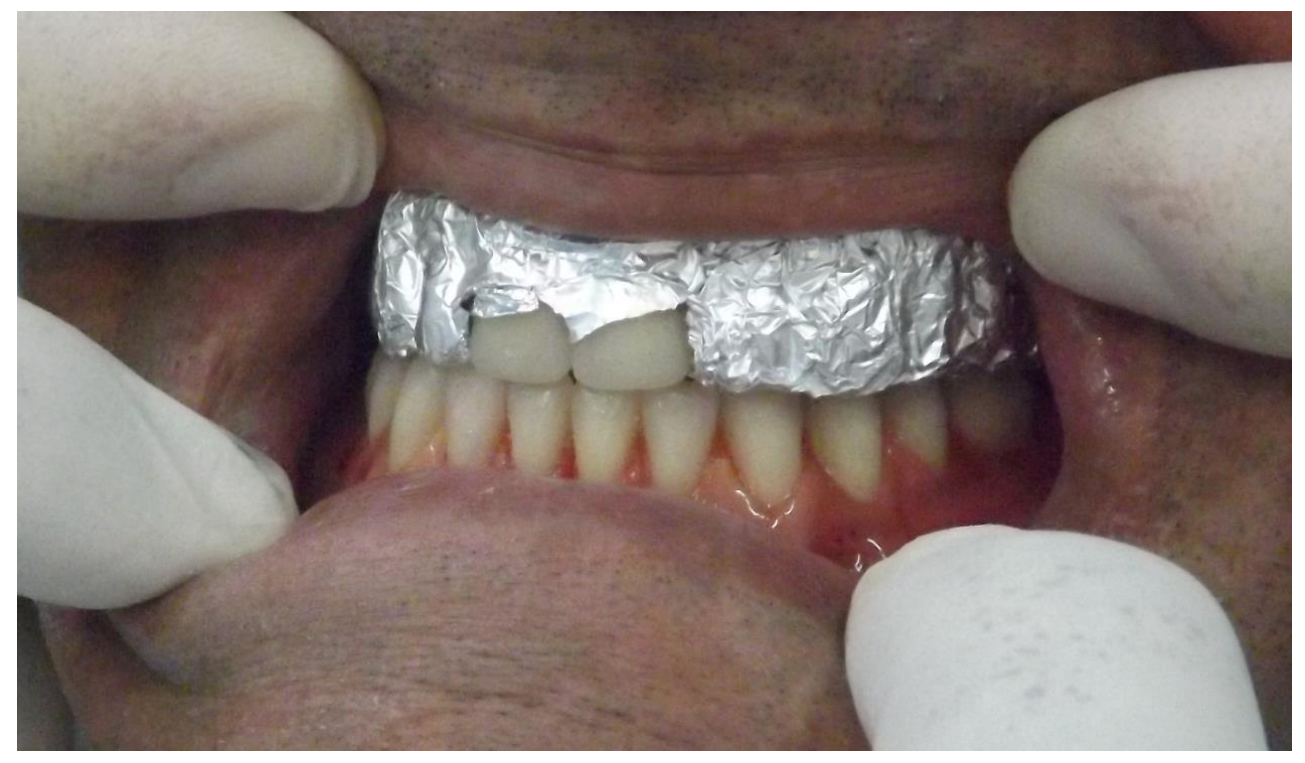

Figura 5: Referente à $10^{\mathrm{a}}$. sessão

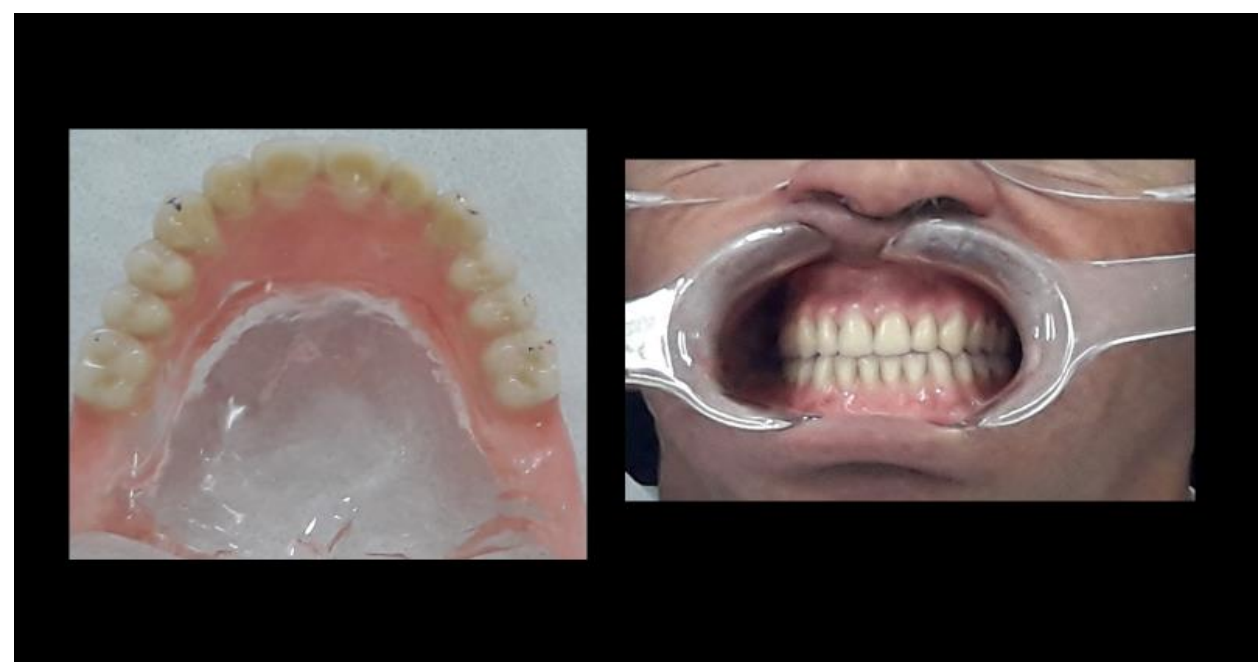

Figura 6: Resultado final na 12 sessão 


\section{Discussão}

A mordida cruzada é um problema de oclusão de etiologia complexa que tem sido tratado ao longo do tempo devido à evolução e ao avanço dos tratamentos ortodônticos que adicionaram crucialmente a aplicabilidade do cuidado em muitas situações. ${ }^{4,11}$ Por essa razão, o ortodontista deve entender cada situação para diagnosticar e analisar corretamente as propriedades de cada tratamento, eliminando riscos e limitações. ${ }^{12} \mathrm{O}$ uso da panaromica aqui foi bem resolvido vez que ela é apta a perceber nuances que ferramentas de imagem tradicionais como o raio x não conseguem detectar. ${ }^{13}$

Vale ser salientado, conforme este estudo que o conceito geral de estética dentária combina vários princípios, incluindo proximidade, similaridade, continuidade e fechamento, viu-se neste caso clinico que a preocupação com todas essas nuances, pois o paciente apresentou problema de maloclusão diagnosticado como mordida cruzada combinado à questão estética. ${ }^{14,15}$

Regis et al. (2016) ${ }^{16}$ revisaram em seu estudo que destacaou a protese total de igual forma a este e passou também por etapas de moldagem clínica, para a obtenção dos modelos de trabalho, garantindo modelos com qualidade superior aos modelos obtidos quando é realizada uma única etapa de moldagem.

Por outro lado, alguns estudos fomentam que não há real necessidade de alguns procedimentos clínicos e laboratoriais considerados essenciais para a confecção das proteses totais. $^{17}$

Viu-se aqui que a protese fixa foi feita em prol de atender aos anseios do paciente devido ao custo e as principais vantagens de corrigir a mordida cruzada

mais rapidamente e menos dispendiosamente. ${ }^{18}$

Nota-se que a preocupação dos profissionais com a acumulação de placa por causa da forma e adaptação da coroa de metal-cerâmica sobre o gingival contorno foi salientada e foram dados ao paciente informações e como proceder para efetuar uma melhor higiene bucal. 4,7,19

Sobre a sessão dez viu-se que Segundo Telles (2014) ${ }^{20}$ nos casos de Classe III, em indivíduos considerados prognatas de perfil côncavo, a melhor relação será a posição de topo a 
topo, com ausência de contatos em RC, para preservar os rebordos. Todavia, será inevitável a desoclusão de dentes anterior em movimento protrusivo.

Um importante fator de adaptação do paciente a uma determinada PT é o controle neuromuscular que o indivíduo desenvolve para manter a prótese estável. Durante a função um movimento lateral que não implique em um movimento vertical, de abertura da mandíbula vai solicitar a atuação de menos fibras musculares durante o movimento, simplificando o controle neuromuscular e facilitando o uso da prótese (TELLES, 2014).

Diante disso viu-se que todos cuidados para que o tratamento fosse um sucesso foram tomados. Assim, como Ning et al. (2005) ${ }^{12}$ que estudou 27 casos de mordida cruzada anterior que foram corrigidos com prótese parcial fixa especialmente concebida e constatou sucesso clínico, funcional e estético em todas as situações. Esse estudo não foi diferente.

Nota-se que o sucesso do tratamento com uma parcial fixa dentadura depende do diagnóstico, tratamento planejamento pelo dentista ou aliado ao técnico de prótese dentária ou higienista, levando em consideração muitas questões e preocupações. Neste caso, a escolha do tratamento foi com base nos princípios da restauração endodôntica com dentes tratados, estética do sorriso e tempo e custo do tratamento. ${ }^{13,19}$

A avaliação apropriada o paciente, o desenho protético e a preparação do dente, bem como boa higiene bucal, são todos necessários para o sucesso. ${ }^{1,8,17}$

Muitos estudos constataram como neste a satisfação dos pacientes com a prótese total. Nos quais pacientes estavam satisfeitos com a qualidade de suas próteses e ainda com sua aparência, relatando melhor qualidade de vida e a qualidade das próteses mostra forte correlação com a satisfação dos pacientes. ${ }^{19,20}$

\section{Conclusões}

Conforme relato de caso foi feita a reabilitação oral de um indivíduo idoso, por meio da confecção de um conjunto de peças protéticas totais na qual foram ainda coletados os dados do prontuário, e realizadas as fotografias. Como resultado, foi obtido uma reabilitação adequada para o caso. 
O resultado obtido por meio deste relato de caso, foi a reabilitação protética adequada de um indivíduo portador de mordida cruzada. Dessa forma, a intenção é divulgar, por meio, acadêmicos e científicos da área odontológica, o sucesso dessa terapia, comprovada pela literatura, no caso utilizado no qual foi reabilitado estética e funcional a dentição do paciente por meio de adequada confecção das próteses adaptada a sua situação clínica.

\section{Referências}

1. Costa Sérgio Carvalho, Carvalho Maria Carmen Fonseca Serpa, Discacciati José Augusto César, Abreu Mauro Henrique Nogueira Guimarães, Viana Érica Leite, Adelário Ana Karoline. Prótese total imediata: devolução do sorriso e da função ao paciente. Arq. Odontol. 2011 Dez ; 47: 106-110.

2. Mallmann Fernanda Hilgert, Toassi Ramona Fernanda Ceriotti, Abegg Claides. Perfil epidemiológico do uso e necessidade de prótese dentária em indivíduos de 50-74 anos de idade, residentes em três 'Distritos Sanitários' de Porto Alegre, Estado do Rio Grande do Sul, Brasil, em 2008. Epidemiol. Serv. Saúde. 2012 Mar; 21( 1 ): 79-88.

3. Franciozi MA, Virmond MCL, Franzolin S, Silva AM, Carvalho J. Influência do tipo de prótese total dupla na função matigatória. Salusvita 2013;32(1):37-45.

4. Trentin LMT, Reginato VF, Maroli A, Borges MTR, Spazzin AO, Bacchi A. Determinação da dimensão vertical de oclusão em prótese total: revisão de literatura e relato de caso clínico. J Oral Invest 2016; 5(1):50-60.

5. Nishimori L, Tomazini TF, Progiante PS, Marson FC, Oliveira e Silva C, Corrêa GO, Correa FDD et al. Estética das próteses flexíveis: relato de caso clínico. Brazilian Journal of Surgery and Clinical Research 2013;5(3):37-40.

6. Carli J, Giaretta B, Vieira R, Linden M, Ghizoni J, Pereira J. Lesões bucais relacionadas ao uso de próteses dentárias removíveis. Salusvita 2013;32(1):103-15.

7. Freitas M, Pacheco G, Zaze C. Seleção de dentes artificiais em próteses odontológicas. Rev Odonto de Araçatuba 2012;33(2):70-74.

8. Bernardo AA, Medeiros MV, Spegel R, Veronez FC V, Trauth KGS. Diagnóstico e planejamento reabilitador em desdentados. Relato de caso. Rev. Odontol. Univ. Cid. São Paulo 2015;27(2):142-499.

9. Montenegro FLB, Ciccuto AF. Montagem em mordida cruzada em próteses totais na Odontogeriatria: relato de caso clínico. Revista Portal de Divulgação 2015;44(5).

10. Laport LBR, Figueira MG, Barbosa MT, Rodrigues CRT, Barbosa OLC. Reabilitação oral com prótese total e prótese parcial removível - relato de caso. Brazilian Journal of Surgery and Clinical Research, 2017;20(1):108-114. 
11. Esteves H, Correia A. Correction of an Anterior Crossbite with a Fixed Partial Denture. JCDA, 2008, 74 (9).

12. Ning JH. Correcting anterior individual crossbite using PFM crowns with porcelain veneer. 6th Annual Scientific Meeting of the IADR Chinese Division; 2005, 24-25.

13. Ahmad I. Anterior dental aesthetics: historical perspective. Br Dent J 2005; 198 (12):737-42.

14. Nuñez MC, Silva DC, Barcelos BA, Leles CR. Patient satisfaction and oral health-related quality of life after treatment with traditional and simplified protocols for complete denture construction. Gerodontology. 2015 Dec; 32(4):247-53.

15. Regis RR, Cunha TR, Della Vecchia MP, Ribeiro AB, Silva-Lovato CH, de Souza RF. A randomised trial of a simplified method for complete denture fabrication: patient perception and quality. J Oral Rehabil. 2013 Jul; 40(7):535-45.

16. Regis RR, Alves CC, Rocha SS, Negreiros WA, Freitas-Pontes KM. The importance of a two-step impression procedure for complete denture fabrication: a systematic review of the literature. J Oral Rehabil. 2016.

17. Oliveira NM, Shaddox LM, Toda C, Paleari AG, Pero AC. Compagnoni MA. Methods for evaluation of masticatory efficiency in conventional complete denture wearers: a systematized review. Oral Health Dent Manag. 2014 Sep; 13(3):757-62.

18. Omar R, Al-Tarakemah Y, Akbar J, Al-Awadhi S, Behbehani Y, Lamontagne P. Influence of procedural variations during the laboratory phase of complete denture fabrication on patient satisfaction and denture quality. J Dent. 2013 Oct; 41(10):852-60.

19. Osterberg T, Carlsson GE, Sundh V, Mellström D. Number of teeth--a predictor of mortality in 70-yearold subjects. Community Dent Oral Epidemiol. 2008 Jun; 36(3):258-68.

20. Telles DM. Prótese total: convencional e sobre implantes. São Paulo: Santos, 2014. 492p.

\section{Como citar este artigo (Formato ABNT):}

CARNEIRO, Rogério Nogueira Celino; PRADO, Jônatas Pereira do; CUNHA, Miguel Arcanjo Porto da; CUHA, Daniela Porto da. Montagem de Prótese Total em Paciente com Mordida Cruzada: Relato de Caso Clínico. . Id on Line Rev.Mult. Psic., 2018, vol.12, n.42, p. 1169-1180. ISSN: 19811179.

Recebido: 25/06/2018;

Aceito: 29/10/2018 